\title{
Update on the Surgical Management of Craniopharyngiomas
}

\author{
Ricardo J Komotar, ${ }^{1}$ Christopher $\mathrm{P}$ Kellner ${ }^{2}$ and Jeffrey $\mathrm{N}$ Bruce ${ }^{3}$ \\ 1. Chief Resident; 2. Junior Resident; 3. Professor of Neurological Surgery, Department of Neurological Surgery, \\ The Neurological Institute of New York, Columbia University Medical Center
}

\begin{abstract}
Despite advances in microsurgical and skull base techniques, the surgical treatment of craniopharyngiomas continues to pose a challenge to surgeons. Controversy continues to exist between proponents of subtotal resection in conjunction with radiotherapy, who argue that this less aggressive approach can yield equivalent control rates with lower morbidity, and others who argue for the superiority of gross total resection. Regardless of whether gross total or subtotal resection is the goal, surgical planning must include a thorough endocrine and neuroophthamological evaluation as well as imaging, and the approach, whether trans-sphenoidal or transcranial, must take into account the nature of the tumour and its location. In addition, optimal management of craniopharyngiomas must consist of individualised and multidisciplinary therapy including an array of subspeciality fields such as endocrinology, neuro-ophthalmology, neuropsychology and radiation oncology.
\end{abstract}

\section{Keywords}

Neurosurgery, craniopharyngioma, craniotomy

Disclosure: The authors have no conflicts of interest to declare.

Received: 25 May 2010 Accepted: 29 June 2010 Citation: European Neurological Review, 2010;5(1):107-12 DOl:10.17925/ENR.2010.05.01.107

Correspondence: Jeffrey N Bruce, Department of Neurological Surgery, The Neurological Institute of New York, Columbia University Medical Center, 710 West 168 th Street, New York, NY 10032, US. E: jnb2@columbia.edu

Craniopharyngiomas are epithelial tumours that arise from embryonic epithelial cells of the craniopharyngeal duct. They account for $1.2-4 \%$ of all primary intracranial neoplasms and $5-10 \%$ of intracranial tumours in children. ${ }^{1}$ Although these tumours are rarely malignant, they often grow adjacent to and distort critical neurovascular structures, particularly the visual pathways and hypothalamus, leading to significant morbidity and mortality. In addition, their sellar or parasellar location poses a challenge to surgical access. However, recent advances in microsurgical and skull base techniques have made resection possible in many patients. ${ }^{2}$

\section{Historical Perspective}

Early attempts at resecting skull base tumours, among them craniopharyngiomas, were limited by a lack of exogenously administered steroids, inadequate visualisation secondary to poor illumination and magnification techniques, poor diagnostic imaging modalities and mortality associated with hypothalamic injury. Despite these extraordinary limitations, early neurosurgeons made significant contributions to the management of craniopharyngiomas. As early as 1912, McArthur addressed the surgical section of the American Medical Association (AMA) regarding surgery of the hypophysis and famously stated: "Iong considered safe from invasion by the most daring surgeon, both because of its almost inaccessible location and because of its intimate relation to life, the question of its feasibility to surgical attack has been answered in the affirmative." ${ }^{\prime 3}$

Due to the proximity of craniopharyngiomas to pituitary tumours, the original surgical approaches were very similarly described as either intracranial or extracranial/transphenoidal. Early intracranial approaches for the resection of pituitary tumours were met with significant failure and high mortality. Following consistent failure of the intracranial approach and given the anatomical belief that these tumours grew downward from the floor of the sella, surgeons often preferred to work through the trans-sphenoidal approach. By contrast, early trans-sphenoidal approaches were more successful. In November 1909, Kanavel devised an infranasal technique that contrasted with other trans-sphenoidal approaches because it passed through the upper part of the nasal cavity. Albert Edward Halstead became the first surgeon to use this technique for the resection of a sellar tumour, notably a craniopharyngioma. Despite success with the trans-sphenoidal approach, not all surgeons were satisfied with the technique. For example, Harvey Cushing initially used the trans-sphenoidal approach, but abandoned it later in his career for the transfrontal approach, likely for better access to suprasellar lesions. Despite improvements in surgical techniques and the occasional success story, the prognosis of hypophyseal tumours in the early 1900s remained dismal.

As knowledge and experience accumulated throughout the early 1900s, intracranial approaches from the side of worse vision improved enough to supplant the trans-sphenoidal approach. By the end of the first half of the 20th century, radical resection was thought to give the best chance of cure, but the high morbidity and mortality associated with radical surgery prevented total resection in the majority of cases. The use of hormonal preparations and 'Roentgen therapy' laid the foundation for future craniopharyngioma treatment. ${ }^{4}$ 
The introduction of corticosteroids as an adjunct to craniopharyngioma management in 1952, the use of post-operative radiation for subtotal resections, the development of microsurgical approaches in the late 1950s and the introduction of computed tomography (CT) scanning in 1972 helped usher in the modern age of craniopharyngioma management. However, with the development of new technologies came new controversies over treatment. Significant debate continues over whether radical resection is superior to partial resection in combination with radiotherapy.

\section{Pre-operative Clinical Considerations}

In order to assist with surgical resection, craniopharyngiomas have been classified by their various locations and extent of growth. Hoffman et al. ${ }^{5}$ use a classification system that delineates tumour location in relation to the sella turcica, optic chiasm and floor of the third ventricle. The tumour classes are pre-chiasmatic, retrochiasmatic, subchiasmatic and intraventricular. Sammi et al. classify craniopharyngiomas based on their vertical projection: grade I (intrasellar or infradiaphragmatic), grade II (occupying the cistern with or without an intrasellar component), grade III (lower half of the third ventricle), grade IV (upper half of the third ventricle) and grade $V$ (reaching the septum pellucidum or lateral ventricles). ${ }^{6}$

Endocrine dysfunction is common and caused by compression of the hypothalamic-hypophyseal axis by the tumour. Children often present with short stature or diabetes insipidus, and hypothalamic symptoms may be present; adolescents may present with delayed or arrested puberty. Cortisol and thyroid hormones (thyroidstimulating hormone [TSH], thyroxine and triiodothyronine) are the most relevant hormones prior to surgery as deficits may have a profound impact on the peri-operative course; however, growth hormone, insulin-like growth factor-1, serum prolactin, folliculestimulating hormone, leuteinising hormone and testosterone and oestradiol in male and female patients, respectively, should be measured to obtain a pre-operative baseline. ${ }^{7}$ Stress-dose exogenous glucocorticoids should be administered due to the risk of hypocortisolaemia in all patients. Oral thyroid hormone replacement should ideally be started one week prior to surgery in order to achieve a euthyroid state. Intravenous (IV) hormone replacement should be reserved for urgent cases.

While $\mathrm{CT}$ is necessary in determining the extent of calcification and locating osseous changes in the skull base, magnetic resonance imaging (MRI) remains the superior modality as it most accurately detects the extent and location of the craniopharyngioma. MRI provides detailed information about the relationship of the tumour to surrounding neurovascular structures, the involvement of the sella, ventricles and posterior fossa, the extent of the tumour in relation to the chiasm and the presence of hydrocephalus, and determines the presence of cystic components that may be amenable to drainage. MR angiography may assist in pre-operative planning by revealing details of the relationship between the tumour and the vasculature.

Visual deficits are also common and result from either direct compression of optic pathways or increased intracranial pressure. Patients may present with decreased visual acuity, diplopia, blurred vision, bitemporal hemianopsia, central scotomas, see-saw nystagmus and blindness. ${ }^{8}$ Visual symptoms are more common in adults, although one study found an equal incidence of visual deficits in children and adults on formal visual field testing. ${ }^{6,9-11}$ Formal visual acuity and visual field testing should be obtained, and fundoscopy should be performed to detect papilloedema or optic atrophy.

Pre-operative hydrocephalus may adversely affect mean survival rate and post-operative outcome. It is recommended to treat patients presenting with clinical signs of hydrocephalus before proceeding with surgical intervention for the tumour. Surgeon preference determines whether or not a cerebrospinal fluid (CSF) diversion procedure is planned prior to surgery or if a shunt is placed intra-operatively. ${ }^{1,6}$

\section{Surgical Evidence}

Despite high morbidity, most neurosurgeons have considered gross total resection (GTR) to be the 'cure' for craniopharyngiomas. ${ }^{12,13}$ However, due to this high morbidity some surgeons advocate a subtotal resection (STR) with concomitant radiotherapy as an alternative therapy. Several studies have found that partial resection and post-operative radiotherapy yield similar disease control rates to GTR with the additional benefit of decreased morbidity. ${ }^{14-20}$

Puget et al. ${ }^{21}$ attempted to identify prognostic indicators in a retrospective cohort of patients with histologically confirmed craniopharyngioma. They used this information to stratify a new prospective cohort of patients to intention to treat for GTR, STR or partial resection. The best indicator of post-operative morbidity was hypothalamic dysfunction measured by pre-operative grade on MRI and hypothalamic function. Patients over five years of age with significant hypothalamic damage secondary to tumour were stratified to STR with radiotherapy; the aim of this approach was to preserve as much hypothalamus as possible. Patients with no hypothalamic dysfunction pre-operatively were stratified to complete excision with re-operation for those with incomplete excision. Patients with moderate hypothalamic damage with tumour abutting or displacing the hypothalamus on MRI underwent GTR, STR or partial resection as deemed appropriate by the operating surgeon. In the prospective series, 23\% of patients underwent GTR, 73\% underwent STR and only one patient (4\%) underwent partial resection. This contradicts the retrospective series in which GTR, STR and PR comprised 50, 36 and $14 \%$ of procedures, respectively, despite intention to treat for GTR. This study demonstrates the possibility for tailored treatment in individuals with craniopharyngioma to minimise hypothalamic damage.

\section{Surgical Approaches}

The surgical approach is predominantly influenced by the tumour location with respect to the sella, chiasm and third ventricle. The ideal approach often varies on a case-by-case basis due to the large number of patient- and tumour-dependent variables incorporated into the decision to use a certain approach. Anterior midline, anterolateral and intraventricular approaches each carry advantages and disadvantages that must be considered in each case (see Table 1).

\section{Anterior Midline Approach}

Historically, there has been significant debate as to whether the trans-sphenoidal or the subfrontal approach is more appropriate for craniopharyngioma resection. ${ }^{15}$ Currently, both approaches are used depending on surgeon preference. The trans-sphenoidal approach is most appropriate for grade I and II (intrasellar and 
Table 1: Operative Approaches

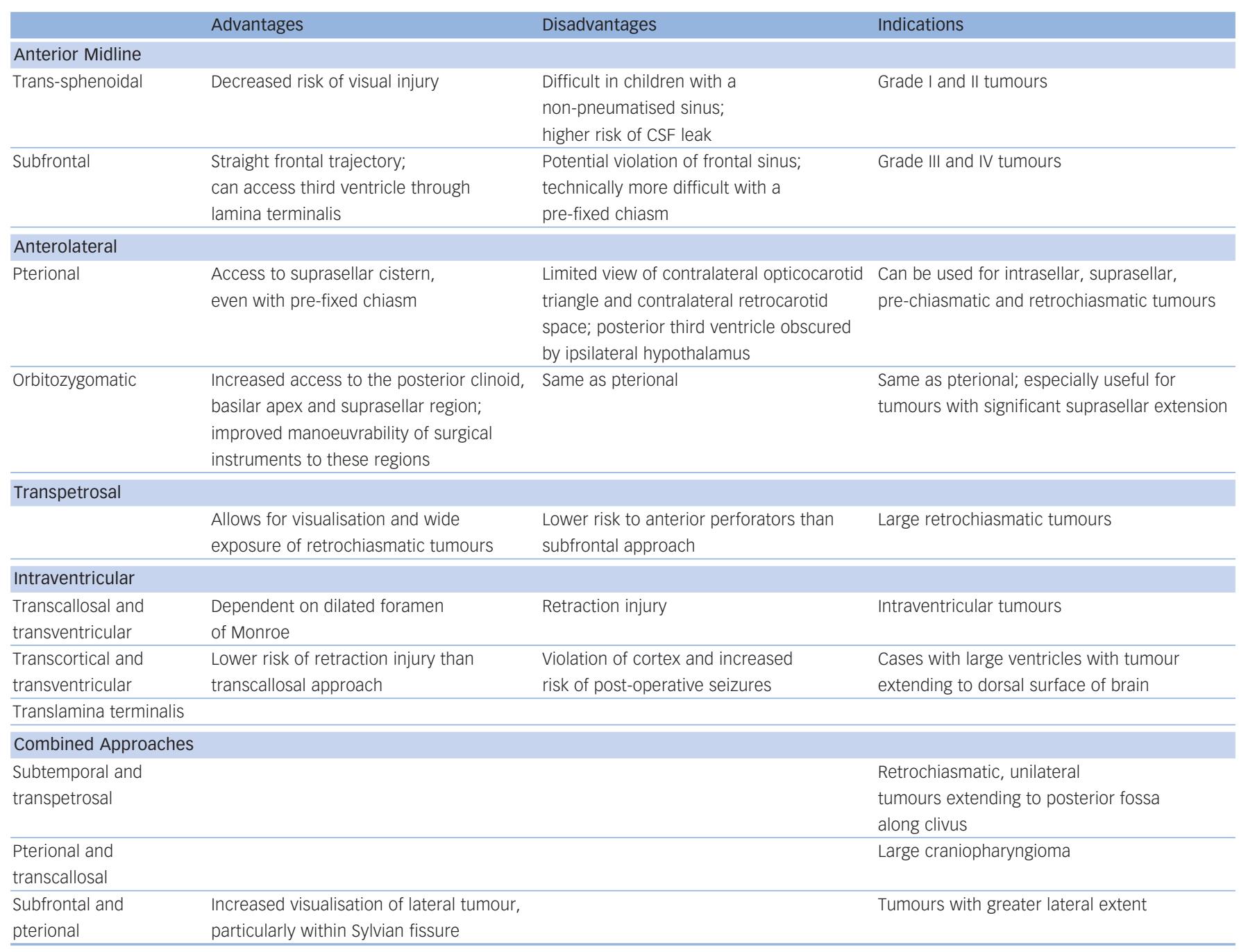

CSF = cerebrospinal fluid.

subdiaphragmatic) tumours because it allows access to the sellar and suprasellar regions with a decreased risk of visual injury. Disadvantages include that it may be difficult in young children with a non-pneumatised sphenoid sinus and the approach carries a higher risk of CSF leak. The subfrontal approach is most appropriate for grade III and IV suprasellar tumours. This approach allows for a straight frontal trajectory with direct access to the anterior third ventricle through the lamina terminalis ${ }^{7}$ and enables pre-chiasmatic dissection of the tumour.

Case series examining this approach have demonstrated that, regardless of size, at least $90 \%$ of all craniopharyngiomas can be removed using the subfrontal and pterional approaches. ${ }^{1,6,10,13}$ Potential complications of the subfrontal approach include the risk of potential violation of the frontal sinus as well as damage to the olfactory tract. In addition, this approach may be technically more complicated in the presence of a pre-fixed chiasm, although this is not an absolute contraindication to the use of the subfrontal approach.

\section{Anterolateral Approach}

The pterional approach is often used in craniopharyngioma resection due to its advantages in accessing the suprasellar cistern, facilitating the resection of intrasellar, suprasellar, pre-chiasmatic and retrochiasmatic tumours. ${ }^{7,13}$ This approach has significant disadvantages; a restricted view of the contralateral opticocarotid triangle, the contralateral retrocarotid space $^{11}$ and the ipsilateral hypothalamic wall makes visualisation of the posterior third ventricle across the lamina terminalis especially difficult. ${ }^{4}$

The orbitozygomatic approach expands on the pterional approach with the addition of removal of the supraorbital rim, zygomatic arch or both, thus increasing access to the posterior clinoid, basilar apex and suprasellar region and improved manoeuvrability of surgical instruments to these regions. ${ }^{7.22-24}$ This approach is useful for lesions with significant suprasellar extension.

\section{Transpetrosal Approach}

The resection of large retrochiasmatic craniopharyngiomas is facilitated by the anteriorly and superiorly oriented surgical corridor made possible by this approach. Conventional anterior approaches carry a risk of injury to the anterior perforators that supply the hypothalamus and chiasm. Injury to the hypothalamus and optic pathways can be minimised by mobilisation of the sigmoid sinus medially, allowing for dissection of a retrochiasmatic tumour under direct visualisation. ${ }^{25,26}$ 


\section{Intraventricular Approaches}

Intraventricular approaches provide access to the craniopharyngioma via the ventricles, which are predominantly accessed through the transcallosal-transventricular approach and the transcorticaltransventricular approach.

The transcallosal-transventricular approach permits access through the lateral ventricle and the foramen of Monroe through an incision in the corpus callosum. In cases where the foramen of Monroe is dilated by a tumour projecting into the lateral ventricle, this approach permits a corridor through which portions of tumour in the third ventricle can be removed. ${ }^{7}$ Retraction of the frontal lobe risks retraction injury, and care must also be taken to avoid damage to the pericallosal arteries, the fornix, the anterior commissure, the choroid plexus, the choroidal arteries and the veins of the wall and floor of the third ventricle. ${ }^{11}$

The transcortical-transventricular approach provides an alternative approach for access to the third ventricle, especially in patients with enlarged lateral ventricles. It lowers the risk to the essentia draining vein of the sagittal sinus and to the pericallosal arteries, but also risks injury to the fornix, thalamus and deep venous structures. The transcortical-transventricular approach also increases the risk of post-operative seizures by exposing the cortex to potential injury. This approach has been reserved for cases with large ventricles and tumour extending to the dorsal surface of the frontal lobe. ${ }^{6.13}$

\section{Translamina Terminalis}

The translamina terminalis approach provides another option to access the third ventricle. It entails opening the lamina terminalis, which is a soft, thin white-matter structure located in the anterior ventricular wall and bound by the optic tracts medially, by the posterior edge of the optic chiasm anteriorly, and by the anterior commissure posteriorly. A pterional or a subfrontal approach can be used to access the lamina terminalis; however, a pterional approach may be more appropriate as the tumour may force the chiasm toward the tuberculum sellae, effectively making it pre-fixed and less amenable to a subfrontal approach. ${ }^{27}$

\section{Combined Approaches}

In some cases, a combination of the above approaches may be employed. The subtemporal-transpetrosal approach can be used for primarily retrochiasmatic unilateral tumours that extend to the posterior fossa along the clivus. ${ }^{26}$ The pterional-transcallosal approach can aid removal of adherent and calcified tumour within the third ventricle. The trans-sphenoidal and transcallosal approaches have also been combined with the pterional approach to allow resection of large craniopharyngiomas. When the transcallosal approach is combined with the pterional approach, intraventricular portions of the tumour should be removed first, with the pterional approach only being performed if basal portions of the tumour remain inaccessible. ${ }^{7}$ The subfrontal-pterional approach has been utilised to remove lateral parts of tumour within the Sylvian fissure. ${ }^{6}$

\section{Endoscopy}

Approaches to the skull base have traditionally been performed via transfacial, transcranial or combined open cranial approaches. Recently, endoscopic approaches to the skull base have been developed and offer a less invasive alternative to open procedures. Whereas traditional microscope-based techniques are used in the transphenoidal approach, endoscopic approaches use rigid and angled endoscopes that allow for up to $360^{\circ}$ visualisation of the skull base without the need for magnification. Furthermore, endoscopic techniques reduce the need for significant brain retraction and the cosmetic deficit associated with open techniques. Depending on the anatomy, different endoscopic approaches have been described utilising various endoscopic corridors (e.g. transnasal, trans-sphenoidal, transethmoidal and transmaxillary). ${ }^{28,29}$ When applied appropriately, endoscopic techniques can achieve high rates of GTR in skull base tumours. Schwartz et al. described a gross total resection rate of $84 \%$ in patients with pituitary tumours for whom GTR was the surgical goal. With regard to patients with craniopharyngiomas, this group reported a GTR rate of $100 \% .{ }^{28}$

Careful patient selection is an essential component of skull base endoscopy. Not all tumours and locations are amenable to this therapeutic strategy. Some general guidelines are that this approach is not appropriate when the lateral extent of the tumour passes more than $1 \mathrm{~cm}$ beyond the lateral limits of the exposure or when the epicentre of the tumour does not lie within the midline.

Furthermore, surgeons must be aware of and realistic about their surgical abilities; for example, although tumour encasing blood vessels is not an absolute contraindication to the endonasal endoscopic approach, pistol grip rather than bayoneted instruments and lack of stereoscopic vision increase the difficulty of dissecting small arteries from the tumour as opposed to the more traditional microscope-guided trans-sphenoidal approach. However, when utilised by a skilled surgeon, endonasal endoscopic approaches have tremendous utility in craniopharyngioma resection, particularly when STR is preferred to GTR and in cases of grade I and II craniopharyngiomas.

\section{Radiation Therapy}

Several alternative therapies can be considered when GTR is not a possibility. Adjuvant radiotherapy may follow STR and be administered via conventional RT, intracavitary radiation or fractionated radiotherapy and as stereotactic radiosurgery. ${ }^{30}$

Although radiotherapy is generally performed as an adjuvant treatment for craniopharyngiomas, there are examples of it being used as a primary treatment modality. Gopalan et al. ${ }^{31}$ performed a meta-analysis of 10 studies to examine the utility, morbidity and mortality of gamma knife surgery (GKS) in craniopharyngioma treatment. Overall tumour control rates were excellent, with singletype tumours demonstrating better control rates than mixed-type tumours. ${ }^{32}$ The mean morbidity rate after GKS was $4 \%$, with a mortality rate of $5 \%$ seen in only one study $(0.05 \%$ overall mortality rate across all studies reviewed). Favourable quality of life outcome was associated with tumours that decreased in size following GKS, while poor outcomes were associated with tumour progression.

GKS involves the delivery of a single high-dose fraction of radiation to a precise target determined by stereotactic neuroimaging-based planning. The precision of targeting and the steep dose fall-off allows for relative sparing of surrounding structures such as the hypothalamus and the visual pathways. A limitation to the use 
of radiotherapy for craniopharyngioma is the radio-sensitivity of the adjacent visual pathways, which are generally restricted to a dose of $<8 \mathrm{~Gy} .{ }^{30}$ Although the optimal dose has been reported to range from 9 to 12Gy, and thus exceeds the dose limit of the optic pathways, therapeutic effect has been reported with a lower marginal dose of 6Gy ${ }^{30,32-34}$ Despite these limitations, GKS can play an important role in reducing recurrence rates when GTR is not feasible.

Stereotactic treatment carries advantages over conventional fractionated radiation therapy by allowing for greater precision and thereby reducing the volume of irradiated brain tissue..$^{35}$ In addition, this modality allows delivery of higher radiation doses with less damage to adjacent neurological structures; for example, the tolerance of the optic chiasm to fractionated stereotactic radiotherapy is reported to be 54Gy/30 fractions compared with a reported 8-9Gy tolerance when delivered as a single fraction. ${ }^{36}$ Minniti et al. ${ }^{37}$ reported a five-year progression-free survival rate of $92 \%$ for fractionated stereotactic radiosurgery compared with 80-90\% for complete excision and 50-60\% for partial resection. However, despite these encouraging survival rates, vasculitis, neuropsychological changes, increased visual deficits and secondary tumours can complicate the use of fractionated stereotactic radiotherapy. ${ }^{38}$

Instillation of intracavitary radiation such as yttrium-90 or phosphorus-32 beta-emitting isotopes has been shown to be an effective primary treatment for cystic craniopharyngiomas. ${ }^{38,39}$ Studies have shown that this therapeutic modality can result in control rates of $96 \%$ for cystic tumours and $88 \%$ for all partially cystic tumours. ${ }^{31}$ Complications of intracavitary radiation include panhypopituitarism, diabetes insipidus and CNS and visual dysfunction, including blindness..$^{31,39,40}$ Although intracavitary radiation is effective for cystic tumours, it is not effective for solid tumours ${ }^{40}$ and carries the possibility of progression of solid portions of the tumour.

Some authors have advocated the combination of GKS and intracavitary irradiation with yttrium-90 or phosphorus-32 isotopes as primary therapy for mixed cystic-solid tumours. ${ }^{38-40}$ This recommendation is based on the observation that solid portions of mixed tumours are more responsive than cystic portions to GKS and that solid tumours and the solid portions of mixed tumours are less responsive than cystic tumours to intracavitary radiation.

\section{Outcomes}

\section{Tumour Recurrence and Survival}

Case series have reported 10-year recurrence-free survival rates for GTR of $74-81 \%,{ }^{15,30,41}$ for partial removal of $41-42 \% \%^{30,42,43}$ and for a combination of surgery and radiotherapy of $83-90 \%{ }^{30,42,43}$ Tenyear recurrence-free survival rates for GTR, STR and STR with radiotherapy have been reported by Karavitaki et al. to be 100 , 38 and $77 \%$, respectively. ${ }^{11}$ Only $18 \%$ of patients in the aforementioned study underwent GTR, in contrast to higher rates of GTR (49-84\%) in other studies. These authors explained their results by stating that the favourable prognosis associated with GTR may have been due to selection bias: the most aggressively growing tumours were only partially removed and subsequently given radiotherapy. Their rationale highlights the limitation faced by studies assessing the efficacy of each treatment modality for craniopharyngiomas (e.g. GTR, STR, STR and adjuvant radiotherapy). The tumours themselves often dictate the indicated treatment, thus the variable efficacies demonstrated in each study may be a function of differences in tumour characteristics rather than solely differences in treatment. This is an important consideration when reviewing treatment outcomes.

Literature values for overall survival range from 80 to $91 \%$ at fiveyear follow-up regardless of treatment modality. ${ }^{43,44}$ The best predictor of survival has been shown to be an absence of recurrence. When recurrences do occur, they do so at mean or median intervals ranging from one to 4.3 years. Treatment of recurrences is difficult, and peri-operative mortality is significantly increased compared with treatment of primary tumour. Scarring from the primary surgery or from radiotherapy decreases the rate of total removal and increases the peri-operative mortality. ${ }^{8,14}$

Radiotherapy with or without surgery is an effective treatment modality for recurrent tumour and significantly prevents further tumour progression. Hakuba et al. ${ }^{26}$ report a 15 -year progressionfree survival of $72 \%$ after radiotherapy, while Stripp et al. ${ }^{8}$ report a 10 -year local control rate of $83 \%$ after radiotherapy.

\section{Morbidity and Management of Complications}

Patients with craniopharyngioma often suffer from endocrine dysfunction and surgical intervention does not seem to reverse existing pre-operative pituitary hormone deficits. The probabilities of $\mathrm{GH}$, follicle-stimulating luteinising hormone (FSH/LH), adrenocorticotropic hormone (ACTH), and TSH deficiencies and diabetes insipidus as 88, 90, 86, 80, and 65, respectively, were reported by Karavitaki et al. ${ }^{11}$ Conditions that should be addressed during the pre-operative evaluation include diabetes insipidus, hypoadrenalism, and hypothyroidism, which have all been shown to increase intra- and post-operative morbidity rates. ${ }^{32}$ Treatment of long-term hormone deficits varies based on the type of deficiency and is best addressed by an endocrinologist. Despite advances in microsurgical technique, hypopituitarism is extremely common and often requires lifelong treatment.

One of the most common presenting symptoms in patients with craniopharyngioma are visual field defects. Visual fields/visual acuity reportedly improved or stabilised in $74 \%$ of patients. ${ }^{45}$ However, despite this, the incidence of long-term major visual field defects has been reported to be $48 \%$ at 10 -year follow-up. ${ }^{11}$ Furthermore, patients can experience a variety of neurological and psychiatric manifestations secondary to their tumour and its treatment. Pereira et al. report the prevalence of short-term memory loss, personality changes, cranial nerve deficits, epilepsy, anosmia and position-dependent vertigo as 40,31, 26, 17, 16 and $12 \%$, respectively. ${ }^{45}$

Despite a progressive reduction in the intraoperative trauma to the hypothalamus due to advances in microsurgical technique, tumours of the sellar region can still result in hypothalamic dysfunction, which can manifest as appetite changes, apathy, sleep disorders and memory deficits. ${ }^{14}$ Hyperphagia and obesity occurs in $26-52 \%$ of post-operative patients. ${ }^{1}$ The sequelae of obesity in craniopharyngioma patients are similar to those seen in the general population; one study recently reported that long-term mortality rates in adult patients with craniopharyngioma are five-fold higher 
than those of the general population. This difference is thought to be largely due to cardiovascular mortality. ${ }^{41}$ Manifestations of hypothalamic dysfunction may significantly affect a patient's psychosocial functioning. One study found that $84 \%$ of adults and $74 \%$ of children treated with surgery were able to live independent lives with social integration and normal professional occupation at a mean follow-up of seven years. ${ }^{41}$ As hypothalamic dysfunction can affect many facets of a patient's biology, management should be multidisciplinary.

\section{Conclusions}

Conclusive treatment for craniopharyngiomas remains a matter of debate. Although GTR yields lower recurrence rates and longer survival, there is a trade-off between efficacy and morbidity. With higher resection rates comes the contrecoup of damaging critical neural and vascular structures in the vicinity of the craniopharyngioma. When GTR is not an option, STR combined with radiotherapy becomes the therapeutic option of choice.

Whether considering GTR or STR, there are many possible surgical approaches to consider. Most importantly, the treatment of craniopharyngiomas is complicated both surgically and medically, necessitating a multidisciplinary approach involving neurosurgery, neurology, endocrinology, ophthalmology and neuropsychology.

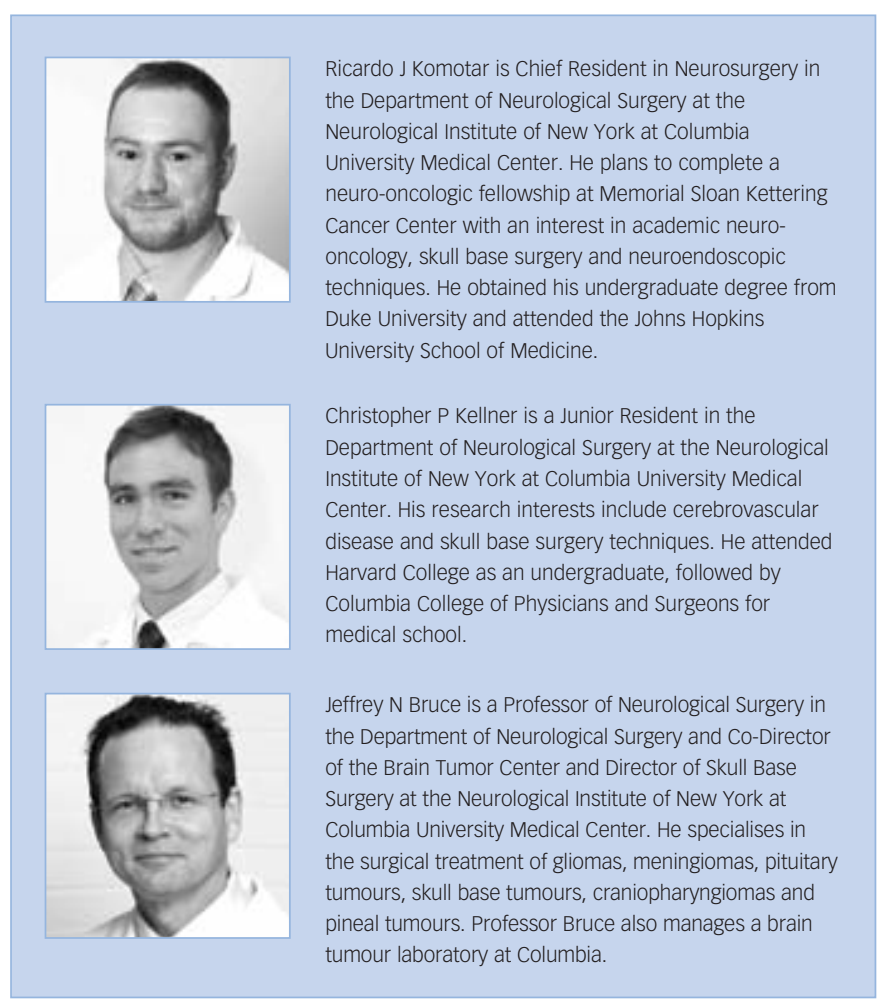

Ricardo J Komotar is Chief Resident in Neurosurgery in the Department of Neurological Surgery at the Neurological Institute of New York at Columbia Uiversity Medical Center. He plans to complete a euro-oncologic fellowship at Memorial Sloan Ketterin Cancer Center with an interest in academic neurocology, skull base surgery and neuroendoscopic echniques. He obtained his undergraduate degree from Duke University and attended the Johns Hopkins School of Medicine. artment of Neurological Surgery at the Neurological Harvard college as an undergraduate followed by Columbia College of Physicians and Surgeons for medical schoo

Jeffrey N Bruce is a Professor of Neurological Surgery in thepartment of Neurological Surgery and Co-Director of the Brain Tumor Center and Director of Skull Base Center. He specialises in the surgical treatment of gliomas, meningiomas, pituitary tumours, skull base tumours, craniopharyngiomas and tumour laboratory at Columbia.
1. Samii M, Bini W, Zentralbl Neurochir, 1991;52:17

2. Komotar RJ, Roguski M, Bruce JN, I Neurooncol, 2009;92:283

3. MCArthur L, JAMA, 2009;58:1912.

4. DiPatri AJ Jr, Prabhu V, Childs Nerv Syst, 2005;21:606,

5. Hoffman HJ, De Silva M, Humphreys RP, et al., J Neurosurg, 1992:76:47.

6. Samii M, Tatagiba M, Neurol Med Chir, 1997:37:141.

7. Liu JK, Cole CD, Kestle JR, et al., Neurosurg Focus, 2005;18:E9.

8. Stripp DC, Maity A, Janss AJ, et al., Int J Radiat Oncol Biol Phys, 2004;58:714

9. Banna M, Hoare RD, Stanley P, et al., J Pediatr, 1973;83:781.

10. Hoff JT, Patterson RH Jr, J Neurosurg, 1972;36:299,

11. Karavitaki N, Brufani C, Warner JT, et al., Clin Endocrinol (Oxf), 2005;62:397.

12. Lapras C, Patet JD, Mottolese $C$, et al., Prog Exp Tumor Res, 1987;30:350.

13. Yasargil MG, Curcic M, Kis M, et al., J Neurosurg, 1990;73:3.

14. De Vile CJ, Grant DB, Kendall BE, et al., J Neurosurg, 1996;85:73.

15. Fischer EG, Welch $K$, Shillito J Jr, et al., J Neurosurg, 1990:73:534

16. Merchant TE, Kiehna EN, Sanford RA, et al., Int J Radiat Oncol Biol Phys, 2002:53:533.
17. Sanford RA, Pediatr Neurosurg, 1994;21(Suppl. 1):39.

18. Shapiro K, Till K, Grant DN, J Neurosurg, 1979;50:617.

19. Scott RM, Hetelekidis S, Barnes PD, et al., Pediatr Neurosurg 1994;21(Suppl. 1):75.

20. Weiss M, Sutton L, Marcial V, et al., Int J Radiat Oncol Biol Phys, 1989;17:1313

21. Puget $\mathrm{S}$, Garnett $\mathrm{M}$, Wray $\mathrm{A}$, et al., J Neurosurg, 2007;106:3.

22. Gonzalez LF, Crawford NR, Horgan MA, et al., Neurosurgery, 2002;50:550

23. Lemole GM Jr, Henn JS, Zabramski JM, et al., J Neurosurg, 2003;99:924.

24. Zabramski JM, Kiris T, Sankhla SK, et al., J Neurosurg, 1998;89:336.

25. Al-Mefty O, Ayoubi S, Kadri PA, Neurosurgery, 2008;62:ONS331.

26. Hakuba A, Nishimura S, Inoue Y, Surg Neurol, 1985;24:405

27. Maira G, Anile C, Colosimo C, et al., Neurosurgery, 2000;47:857.

28. Schwartz TH, Fraser JF, Brown S, et al., Neurosurgery, 2008:62:991.

29. Schwartz TH, Anand VK, Clin Neurosurg, 2007;54:226

30. Duff JM, Meyer FB, IIstrup DM, et al., Neurosurgery, 2000:46:291

31. Gopalan R, Dassoulas K, Rainey J, et al., Neurosurg Focus, 2008:24:E5.
32. Chung WY, Pan HC, Guo WY, et al., Stereotact Funct Neurosurg, 1998:70(Suppl. 1):139.

33. Kobayashi T, Kida Y, Mori Y, et al., J Neurosurg, 2005;103:482

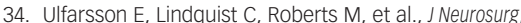
2002;97:613.

35. Kalapurakal JA, Childs Nerv Syst, 2005;21:808.

36. Tishler RB, Loeffler JS, Lunsford LD, et al., Int J Radiat Oncol Biol Phys, 1993;27:215

37. Minniti G, Saran F, Traish D, et al., Radiother Oncol, 2007;82:90

38. Albright AL, Hadjipanayis CG, Lunsford LD, et al., Childs Nerv Syst, 2005;21:649.

39. Voges J, Sturm V, Lehrke R, et al., Neurosurgery, 1997;40:263.

40. Hasegawa T, Kondziolka D, Hadjipanayis CG, et al. Neurosurgery, 2004;54:813.

41. Bulow B, Attewell R, Hagmar $L$, et al., J Clin Endocrinol Metab, 1998;83:3897.

42. Hetelekidis S, Barnes PD, Tao ML, et al., Int I Radiat Oncol Biol Phys, 1993;27:189.

43. Rajan B, Ashley S, Gorman C, et al., Radiother Oncol, 1993;26:1.

44. Symon L, Sprich W, J Neurosurg, 1985;62:174

45. Pereira AM, Schmid EM, Schutte PJ, et al., Clin Endocrinol, 2005:62:197. 